\title{
Zone-center phonon frequencies for graphite and graphite intercalation compounds: Charge-transfer and intercalate-coupling effects
}

\author{
C. T. Chan, K. M. Ho, and W. A. Kamitakahara \\ Ames Laboratory, Iowa State University, Ames, Iowa 50011
}

(Received 8 June 1987)

\begin{abstract}
The frequencies of several zone-center vibrational modes in graphite have been calculated from first principles, with results in very good agreement with experiment. By studying the effect of charge transfer and lattice-constant change on $E_{2 g}$-mode frequencies, we find that the systematic stage dependences of both Raman- and infrared-active modes in graphite intercalation compounds can be understood by introducing a coupling to the intercalate of unconventional form.
\end{abstract}

The zone-center phonon frequencies of graphite intercalation compounds (GIC's), as measured by Raman, infrared (ir), and neutron spectroscopy, have provided many useful insights into the physics of these materials. ${ }^{1}$ In particular, the stage dependence of Raman-active $E_{2 g_{2}}$ modes shows ${ }^{2,3}$ that bounding graphitic layers (those immediately bounding an intercalate layer in a GIC) have frequencies distinct from those of interior graphitic layers (those not adjacent to intercalate layers), with the relative Raman intensities depending on stage number $n$ in a way which implies that the charge transferred from intercalate to graphitic host resides primarily on the bounding layers. The frequencies (as opposed to the intensities) of the bounding-layer (BL) and interior-layer (IL) modes also exhibit systematic dependences ${ }^{2}$ on $1 / n$, which are distinct for donors and acceptors, but nearly universal within each group, offering a tantalizing clue that the phonon frequencies might contain additional valuable information about the physics of GIC's, if only a sound theoretical basis existed. Leung, Dresselhaus, and Dresselhaus ${ }^{4}$ and Al-Jishi and Dresselhaus ${ }^{5}$ have addressed this problem using Born-von Kármán models with empirical force constants. The purpose of the present calculation is to provide a more microscopic understanding of the zone-center frequencies by (i) performing first-principles calculations which separate out the effects of charge transfer and latticeconstant change, and (ii) constructing a simple phenomenological model which incorporates the first-principles results, but also takes coupling to the intercalate ions or molecules into account.

Our starting point is a series of fully self-consistent electronic-structure calculations on pure graphite within the local-density formalism, ${ }^{6}$ using $a b$ initio pseudopotentials ${ }^{7}$ and an $a b$ initio linear combination of atomic orbitals (LCAO) method. ${ }^{8}$ Results for the in-plane $\mathrm{C}-\mathrm{C}$ bond length $d_{\mathrm{C}-\mathrm{C}}$, the elastic constant $\mathrm{C}_{11}+\mathrm{C}_{12}$, and some zone-center phonon frequencies, shown in Table I, are in in excellent agreement with experiment, considering that the crystal structure, atomic number, and atomic mass are the only inputs. Each phonon calculation involved a series of total-energy evaluations while imposing a static phononlike distortion ("frozen-phonon" method $^{9}$ ), involving band-structure computation at over $100 k$ points in the irreducible Brillouin zone. The $E_{2 g}$ frequency in Table I was calculated with the graphitic planes in $A A$ stacking, rather than the real $A B$ stacking, because the stacking sequence should have a miniscule effect on this in-plane mode. The out-of-plane $A_{2 u}$-mode frequencies in $A A$ and $A B$ stacking are nearly the same within the computational accuracy of a few $\mathrm{cm}^{-1}$, consistent with the fact that this is also an intralayer mode, governed mainly by strong intralayer $\mathrm{C}-\mathrm{C}$ bonding. In contrast, the frequency of the completely interlayer $B_{1 g_{1}}$ mode is higher in $A A\left(180 \mathrm{~cm}^{-1}\right)$ than in $A B(133$ $\mathrm{cm}^{-1}$ ) stacking. The ratio $180 / 133$ is nearly $\sqrt{2}$, which is expected if only nearest-neighbor interatomic forces operate between layers.

We next describe first-principles calculations of the effect of charge transfer on the in-plane zone-center $E_{2 g}$ mode frequency for GIC's. In order to isolate the effect of charge transfer, the intercalate is simulated by point ions with charge $Z, Z \ll 1$, which are centered above $C$ hexagons, midway between two graphitic layers. These structureless ions keep the system neutral by balancing the electronic charge donated to or depleted from the graphitic planes. Previous use of this model ${ }^{10}$ demonstrated that charge transfer is the dominant factor in determining $d_{\mathrm{C}-\mathrm{C}}$ changes in GIC's, providing a convenient method of determining charge transfer by a simple lattice-constant measurement. In Fig. 1(a), we see that charge donation to the graphitic planes causes an almost linear decrease in frequency, whereas charge depletion causes a much weaker and nonlinear stiffening. The lattice constants were

TABLE I. First-principles calculations for properties of graphite, compared with experiment. $A A$ rather than $A B$ stacking was used for $E_{2 g}$, but not for $A_{2 u}$ or $B_{1 g_{1}}$ modes. Experimental data are from Table 2.1 of Ref. 2.

\begin{tabular}{lcc}
\hline \hline & Theory & Experiment \\
\hline In-plane $C-C$ bond length $(\AA)$ & 1.419 & 1.421 \\
$C_{11}+C_{12}\left(10^{11}\right.$ dyn cm $\left.^{-2}\right)$ & 125 & 124 \\
$\omega\left(E_{2 g}\right)\left(\mathrm{cm}^{-1}\right)$ & 1598 & 1582 \\
$\omega\left(A_{2 u}\right)\left(\mathrm{cm}^{-1}\right)$ & 839 & 868 \\
$\omega\left(B_{1 g_{1}}\right)\left(\mathrm{cm}^{-1}\right)$ & 133 & 128 \\
\hline \hline
\end{tabular}





FIG. 1. First-principles calculations of the $E_{2 g}$ mode frequency as functions of (a) charge transfer, and (b) lattice-constant charge.

kept at those of pristine graphite to compute only the direct effect due to charge transfer. However, charge transfer to the graphitic host will cause a lattice-constant change (calculated quantitatively in Ref. 10), in turn changing $\omega\left(E_{2 g}\right)$. Calculations of this indirect effect [Fig. 1(b)] with $z=0$ show an almost linear decrease of frequency with increasing lattice parameter yielding a mode Gruneisen parameter of 1.87 , in rather good agreement with experiment ${ }^{11}(1.66 \pm 0.13)$. Because it is generally accepted ${ }^{12}$ that the transferred charge for higherstage GIC's $(n \geq 3)$ is localized on graphitic BL's, we henceforth assume that the direct effect of charge transfer on in-plane mode frequencies is confined to the BL's. We also assume that the effect of lattice-constant change is felt by all layers, assuming, in accord with experiment, ${ }^{13}$ that IL's and BL's have the same lattice constant, sharing the charge-transfer-induced stress.

It is useful at this point to review the observed systematic stage dependences of the Raman-active $E_{2 g_{2}}{ }^{-}$ mode ${ }^{14}$ and ir-active $E_{1 u}$-mode frequencies (Figs. 2 and 3). The $E_{2 g_{2}}$ frequencies increase with inverse stage number $1 / n$ (or intercalate concentration) for acceptors, but decrease for donors. This is consistent with the trends of Fig. 1, which show, e.g., for donors, that both charge donation and in-plane expansion soften the mode. However, it is clear that there are other features that cannot be explained by the charge-transfer effects of Fig. 1 alone. For both donors and acceptors, the Raman-active BL $E_{2 g_{2}}$ modes have higher frequencies than the corresponding IL modes. This is unexpected from charge-transfer effects

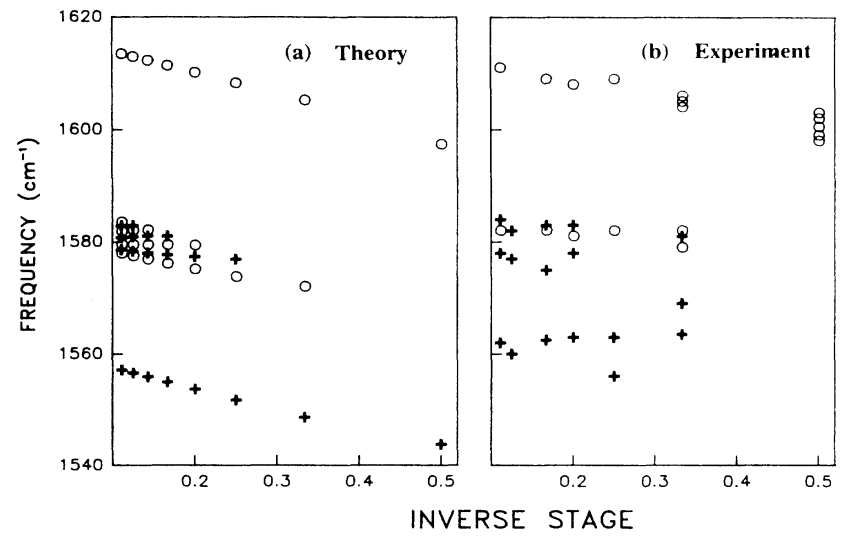

FIG. 2. (a) Theoretical and (b) experimental stage dependences of the Raman $(O)$ and ir $(+)$ modes for donor GIC's. In (b), the Raman data for Rb, Cs, K, Li are from Table 2.5 of Ref. 2, while the ir data for Rb, K are from Fig. 3 of Ref. 4.

- the BL mode should have a higher frequency in acceptors but a lower frequency in donors. Moreover, for the ir-active $E_{1 u}$ mode, the BL mode is lower in frequency than IL modes for both donors and acceptors. This cannot be explained by changes in the intralayer force constant, since the in-plane atomic displacements for $E_{1 u}$ and $E_{2 g_{2}}$ modes are the same, differing only in the relative phase of atomic displacements in adjacent planes. Hence, we conclude that other effects due to the intercalate must influence the $E_{2 g_{2}}$ and $E_{1 u}$ frequencies.

We now discuss a model in which these effects are taken into account in the simplest possible way. We emphasize that our goal is not to produce a set of parameters to best fit the experimental data, but rather to provide a global understanding of the behavior of the zone-center modes. We model the GIC system by an infinite chain of coupled harmonic oscillators, each representing the in-plane, zone-center phonon of a graphitic layer, with a force con-

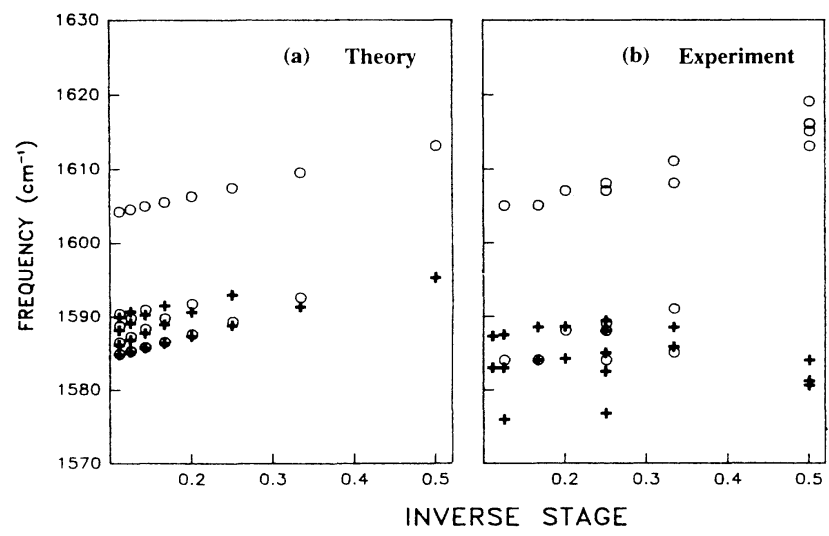

FIG. 3. (a) Theoretical and (b) experimental stage dependences of the Raman $(O)$ and ir $(+)$ modes in acceptor GIC's. In (b), the Raman data for $\mathrm{AsF}_{5}, \mathrm{FeCl}_{3}, \mathrm{AlCl}_{3}, \mathrm{SbCl}_{5}$ are from Table 2.5 of Ref. 2, while the ir data for $\mathrm{FeCl}_{3}, \mathrm{AlCl}_{3}$ are from Fig. 4 of Ref. 4. 
stant $c$ for an IL, or $b$ for a BL. Localization of the transferred charge on BL's implies that for $n \geq 2$, the charge on the BL's stays nearly constant. Hence, the direct effect of charge transfer on the value of $b$ is the same for $n \geq 2$ and can be determined from our calculated results [Fig. 1(a)], if we know the amount of charge transfer. Since different stages have different lattice constants, both $c$ and $b$ have stage dependences which we can infer from our calculation of the dependence of the frequency on the lattice constant [Fig. 1(b)]. The oscillators representing IL's are coupled together by a weak force constant $k$ that gives the $6-\mathrm{cm}^{-1}$ splitting of the Raman and ir modes in pure graphite.

The existence of systematic frequency dependence on stage number indicates that the intercalate effect cannot depend strongly on the chemical or structural detail of the intercalate. We propose that it arises from an effective coupling between bounding layers straddling an intercalate layer, as represented by the last term of the Hamiltonian

$$
H=\sum_{j, i}\left(\frac{m}{2} \dot{u}^{2}(j, i)+\frac{h}{2} u^{2}(j, i)\right)+\sum_{j, i=1}^{n-1} \frac{k}{2}[u(j, i+1)-u(j, i)]^{2}+\sum_{j} \frac{g}{2}[u(j+1,1)+u(j, n)]^{2}
$$

where $n$ is the stage, $u(j, i)$ are displacements of oscillator $i$ in unit cell $j$, and $h=b$ or $h=c$, depending on whether $i$ labels a BL or IL. The coupling represented by the last term, involving a sum of displacements, gives rise to interactions that are not standard two-body Born-von Kármán forces. Its physical meaning becomes more obvious if we write

$$
g(u+v)^{2} / 2=g_{1}\left(u^{2}+v^{2}\right)+g_{2} u v,
$$

where $g_{1}=g / 2$ and $g_{2}=g$. When the $C$ atoms vibrate about their equilibrium positions, they squeeze the intercalate atoms or molecules in their immediate vicinity, causing an increase in frequency for both donor and acceptor GIC's, which is represented by $g_{1}$. The coupling $g_{2}$ allows for a stronger stiffening effect for the Ramanactive (even) mode than for the ir-active (odd) mode. For the even mode, the atomic displacements in two adjacent BL's are in phase, so that the $\mathrm{C}$ atoms above and below the intercalate squeeze it simultaneously, whereas for the odd mode, the atomic displacements are out of phase so that when the $\mathrm{C}$ atoms in the layer above are squeezing the intercalate, those in the layer below are retreating from it. There is no fundamental reason why one should have $g_{2}=2 g_{1}$, beyond allowing us to consider this unconventional steric effect using only a single adjustable parameter. Imposing periodic boundary conditions on Eq. (1) yields an $n \times n$ dynamical matrix whose eigenvalues are the squared phonon frequencies. We restrict our model to stages $n \geq 2$, because all BL's are then essentially the same.

For alkali-metal donor compounds, we have a fairly simple situation in which the intercalates consist of spherical atoms with, except for $\mathrm{Li}$, the formula $M \mathrm{C}_{12 n}$ for stage $n \geq 2$. Hence, one value of the coupling parameter $g$ may suffice for all of them. Moreover, charge transfer is basically complete for these GIC's, i.e., $\frac{1}{24}$ of an electron is donated to each BL C atom and hence $b$ [as inferred from Fig. 1(a)] should be $-1560 \mathrm{~cm}^{-1}$ at the equilibrium lattice constant of graphite. The stage dependence of $b$ and $c$ can be deduced immediately by combining the calculated dependences of frequency on $d_{\mathrm{C}-\mathrm{C}}$ and of $d_{\mathrm{C}-\mathrm{C}}$ on stage. The latter can be calculated from the charge transfer and the known stoichiometry of the GIC, as described in detail in Ref. 10. Using a value of $\mathrm{g} / \mathrm{m}=88928 \mathrm{~cm}^{-2}$, we obtain the frequencies plotted against $1 / n$ in Fig. 2(a) for $n=2$ to 9 . Examining Fig. 2(a), we note first that all frequencies decrease as a function of $1 / n$ due to the increase of $d_{\mathrm{C}-\mathrm{C}}$. There is a group of modes (both even and odd, split slightly by the force constant $k$ ) clustered around $1580 \mathrm{~cm}^{-1}$ which have eigenvectors distributed mainly on the IL's, corresponding to the IL modes observed in Raman and ir spectroscopies. There is an even mode split off to a higher frequency, $\sim 1610 \mathrm{~cm}^{-1}$, and an odd mode split off to low frequency, $-1550 \mathrm{~cm}^{-1}$. The eigenvectors of these modes clearly identify them as the BL modes observed in experiments. The stage dependence for the high-frequency even mode compares well with those of the BL mode observed in Raman experiments. The lowfrequency odd mode in the model is a few $\mathrm{cm}^{-1}$ 's lower than the range of frequencies observed for BL ir modes. Better agreement can be obtained if one chooses to use two adjustable parameters (i.e., vary $g_{1}$ and $g_{2}$ independently) instead of one.

Acceptors offer a more complicated picture as the shape, size, arrangement pattern, and in-plane density of the intercalate are highly variable. Nevertheless, we find that both the observed Raman and ir frequencies can be discussed within our model. The stage dependence of the force constants $b$ and $c$ are estimated from the combined knowledge of the measured stage dependence of $d_{\mathrm{C}-\mathrm{C}}$ for $\mathrm{D}_{2} \mathrm{SO}_{4}$ intercalated graphites ${ }^{10}$ and the dependence of frequency on $d_{\mathrm{C}-\mathrm{C}}$ from the present calculation [Fig. 1(b)]. A value of $\mathrm{g} / \mathrm{m}=28638 \mathrm{~cm}^{-2}$ then gives the frequencies shown in Fig. 3(a). The acceptor modes stiffen as a function of $1 / n$ because of $d_{\mathrm{C}-\mathrm{C}}$ contraction. There is an even mode split off to higher frequencies, which, on the basis of the oscillator eigenvectors, can be identified with the Raman-active BL modes. In contrast to donors, the theory with a single parameter [as in Eq. (1)] does not give distinct $\mathrm{BL}$ odd modes that split off below the IL modes $\left(\sim 1585 \mathrm{~cm}^{-1}\right)$. The presence of the lowfrequency BL odd modes in donors arises from the modesoftening effect of charge donation in donors, but for acceptors, BL odd modes with frequencies lower than 1580 $\mathrm{cm}^{-1}$ exist only if $g_{1}$ and $g_{2}$ are varied independently.

Our generic theory agrees better with the observed Raman modes than the far less systematic ir modes. It is gratifying that salient experimental features (e.g., mode softening for donors, mode stiffening for acceptors, highfrequency $\mathrm{BL}$ even modes for both donors and acceptors) 
are reproduced in the theory once our first-principles results are augmented by an effective intercalate coupling with a single parameter for each class of GIC's. In summary, we have shown that unlike changes in the bond length $d_{\mathrm{C}-\mathrm{C}}$, which are almost completely determined by charge transfer, ${ }^{10}$ changes in the in-plane phonon frequencies depend on both charge transfer and coupling to the intercalate.
We thank Dr. P. C. Eklund, Dr. G. W. Lehman, and Dr. B. N. Harmon for stimulating discussions. The Ames Laboratory is operated by Iowa State University for the U.S. Department of Energy (USDOE) under Contract No. W-7405-ENG-82. Support for this work, including a grant of Cray computer time at the Lawrence Livermore Laboratory, was provided by the Director for Energy Research, Office of Basic Energy Sciences, USDOE.
${ }^{1}$ M. S. Dresselhaus and G. Dresselhaus, Adv. Phys. 30, 139 (1981), and references therein.

${ }^{2}$ M. S. Dresselhaus and G. Dresselhaus, in Light Scattering in Solids III, edited by M. Cardona and G. Guntherodt, Topics Applied Physics, Vol. 51 (Springer-Verlag, New York, 1982), Chap. 2, and references therein.

${ }^{3}$ S. A. Solin, Mater. Sci. Eng. 31, 153 (1977).

${ }^{4}$ S. Y. Leung, G. Dresselhaus, and M. S. Dresselhaus, Synth. Met. 2, 89 (1980).

${ }^{5} \mathrm{R}$. Al-Jishi and G. Dresselhaus, Phys. Rev. B 26, 4523 (1982).

${ }^{6}$ See, e.g., Theory of the Inhomogeneous Electron Gas, edited by N. H. March and S. Lundqvist (Plenum, New York, 1983).

${ }^{7}$ D. R. Hamann, M. Schlüter, and C. Chiang, Phys. Rev. Lett. 43, 1494 (1979).

${ }^{8}$ J. R. Chelikowsky and S. G. Louie, Phys. Rev. B 29, 3470
(1984); C. T. Chan, D. Vanderbilt, and S. G. Louie, ibid. 33, 2455 (1986)

${ }^{9}$ See, e.g., S. G. Louie, in Electronic Structure, Dynamics, and Quantum Structural Properties of Condensed Matter, edited by J. T. Devreese and P. V. Camp (Plenum, New York, 1985), p. 335.

${ }^{10}$ C. T. Chan, W. A. Kamitakahara, K. M. Ho, and P. C. Eklund, Phys. Rev. Lett. 58, 1528 (1987).

${ }^{11}$ N. Wada, Phys. Rev. B 24, 1065 (1981).

${ }^{12}$ See, e.g., N. A. W. Holzwarth, S. G. Louie, and S. Rabii, Phys. Rev. B 28, 1013 (1983).

${ }^{13}$ D. E. Nixon and G. S. Parry, J. Phys. C 2, 1732 (1969).

${ }^{14} \mathrm{We}$ have used $E_{2 g}$ rather than $E_{2 g_{2}}$ to label the mode in the theoretical results because $A A$ rather than $A B$ stacking for graphite is used to compute the frequency of this particular mode. 